\title{
Investigation of the Neural Control of Cough and Cough Suppression in Humans Using Functional Brain Imaging
}

\author{
Stuart B. Mazzone, ${ }^{1}$ Leonie J. Cole, ${ }^{2}$ Ayaka Ando, ${ }^{2}$ Gary F. Egan, ${ }^{2,3}$ and Michael J. Farrell ${ }^{2,3}$ \\ ${ }^{1}$ The School of Biomedical Sciences, University of Queensland, St. Lucia, Queensland 4072, Australia, and ${ }^{2}$ Florey Neurosciences Institutes and ${ }^{3}$ The Centre \\ for Neuroscience, University of Melbourne, Victoria 3010, Australia
}

Excessive coughing is one of the most common reasons for seeking medical advice, yet the available therapies for treating cough disorders are inadequate. Humans can voluntarily cough, choose to suppress their cough, and are acutely aware of an irritation that is present in their airways. This indicates a significant level of behavioral and conscious control over the basic cough reflex pathway. However, very little is known about the neural basis for higher brain regulation of coughing. The aim of the present study was to use functional brain imaging in healthy humans to describe the supramedullary control of cough and cough suppression. Our data show that the brain circuitry activated during coughing in response to capsaicin-evoked airways irritation is not simply a function of voluntarily initiated coughing and the perception of airways irritation. Rather, activations in several brain regions, including the posterior insula and posterior cingulate cortex, define the unique attributes of an evoked cough. Furthermore, the active suppression of irritant-evoked coughing is also associated with a unique pattern of brain activity, including an involvement of the anterior insula, anterior mid-cingulate cortex, and inferior frontal gyrus. These data demonstrate for the first time that evoked cough is not solely a brainstem-mediated reflex response to irritation of the airways, but rather requires active facilitation by cortical regions, and is further regulated by distinct higher order inhibitory processes.

\section{Introduction}

Coughing in response to airway irritation or accumulated secretions is essential for protecting the respiratory system. However, patients with excessive or chronic cough experience a range of physical, social, and emotional deficits that contribute to a severe reduction in their quality of life and predispose them to depressive illnesses (McGarvey et al., 2006; Brignall et al., 2008). The act of coughing involves a complex neural network that remains mostly undescribed. Coughing can be initiated by irritation of sensory nerves in the airways via a reflex response that is not dependent on contributions from supramedullary structures (Baekey et al., 2001; Ohi et al., 2004; Mazzone, 2005). This basic network includes subsets of vagal sensory neurons that respond to chemical or mechanical irritation and provide excitatory drive to the brainstem cough pattern generator (Shannon et al., 2000; Baekey et al., 2001).

Overlaid onto this basic reflex is a series of more complicated central and peripheral regulatory inputs. For example, in humans coughing can be voluntarily initiated via processes that involve activations of the sensorimotor cortex, insula, anterior cingulate cortex, supplementary motor area, thalamus, and basal ganglia

Received Aug. 31, 2010; revised Dec. 20, 2010; accepted Dec. 23, 2010.

This work was supported by National Health and Medical Research Council (NHMRC) of Australia Grant 566734. S.B.M. and G.F.E. hold current NHMRC Fellowship Grants 454776 and 400317. M.J.F. is a C. R. Roper Fellow. We acknowledge the technical expertise of Michael Kean of the Children's Magnetic Resonance Imaging Centre (Melbourne, Australia).

Correspondence should be addressed to Dr. Stuart B. Mazzone at the above address. E-mail: s.mazzone@uq.edu.au.

DOI:10.1523/JNEUROSCI.4597-10.2011

Copyright $\odot 2011$ the authors $\quad 0270-6474 / 11 / 312948-11 \$ 15.00 / 0$
(Simonyan et al., 2007). Presumably, one or more of these brain regions provide "top-down" drive to the cough pattern generator to initiate a voluntary cough motor pattern. Similarly, humans can suppress their cough, although this brain circuitry has not been described. Airway irritations also lead to the perception of the urge to cough, a clinical symptom that is analogous to an itch and may underlie behavioral coughing in airways diseases (Davenport, 2009). We have described urge-to-cough brain activations, many of which overlap with the cortical and subcortical correlates of pain (Mazzone et al., 2007, 2009a). Indeed, like pain, airways irritation evokes activations in areas associated with sensory, motor, cognitive, affective, and motivational responses (Mazzone et al., 2009a). Deciphering the roles of higher order processes in excessive coughing in disease represents a significant challenge.

Cough research has been hindered by the unpredictable variability in the reflex. Not all animals cough in response to airways irritation and cough evoked by many stimuli is absent during general anesthesia (Nishino et al., 1988; Canning et al., 2004; Mazzone et al., 2009b). One approach for identifying brain regions involved in coughing in humans is to use an event-related functional magnetic resonance imaging (fMRI) protocol that measures blood oxygen level-dependent (BOLD) responses, representative of neural activity, during periods of airway irritation and/or voluntary or reflex-evoked coughing. However, separating neural responses specific to cough from those associated with generating ongoing respiratory rhythm, modifications in respiration, and any resultant changes in blood gases requires careful design and analysis (Birn et al., 2008). Studies can also be confounded when tasks involve correlated head movements, as can 
be the case with coughing. The aim of this study was to overcome these issues by implementing analytical techniques that incorporate respiratory response functions and head movement modeling to reveal the central circuitry involved in the facilitation and suppression of cough reflex pathways in humans. We hypothesize that reflex and voluntary control of coughing will be associated with supramedullary brain activation that reflect cough facilitation and cough suppression.

\section{Materials and Methods \\ Subjects}

Twenty-one people gave written informed consent to participate in the study, which was approved by the Melbourne Health Human Research Ethics Committee (Australia). Before inclusion, all subjects were screened via telephone interview to exclude conditions that could potentially alter cough sensitivity, including chronic respiratory or neurological disease or acute respiratory infection. All subjects were nonsmokers and were not taking any medications likely to influence cough sensitivity or fMRI BOLD responses at the time of testing. Data from one subject who was unable to comply with the experimental protocol, and another subject with excessive $(>3 \mathrm{~mm}$ ) event-related head motion, were excluded from group analyses. Thus, a total of 19 subjects ( 7 females; mean age, $24.9 \pm$ 8 years) were included in the final analysis (see Table 1).

\section{Inhaled challenges}

The urge to cough was evoked via the inhalation of capsaicin solution, which was prepared in doubling doses from 0.25 to $125 \mu \mathrm{M}$ as previously described (Mazzone et al., 2007). Normal saline solution $(0.9 \% \mathrm{NaCl})$ was used as a comparison stimulus. Stimuli (capsaicin or saline solution) were delivered using a custom-designed apparatus consisting of a modified face mask attached via removable tubing to three jet nebulizers (RapidFlo; Allersearch). Each nebulizer was routed in parallel to its own dedicated shut-off valve on a main switchboard, which also contained a fourth valve for the passive exhaust of air. All four valves were in turn connected in series to an air compressor (flow rate, $0.42 \mathrm{ml} / \mathrm{min}$ ) (Liberty Healthcare) during the psychophysical testing session and to medical air (flow rate, $0.5 \mathrm{ml} / \mathrm{min}$ ) during the brain imaging session. Experimental visual cues during fMRI scanning were delivered using Presentation software (Neurobehavioral Systems).

\section{Procedures}

Psychophysical session: cough threshold determination

For each subject, the concentration of inhaled capsaicin solution required to evoke two or more coughs was determined using the method of limits and denoted as the $\mathrm{C} 2$ threshold. To simulate the conditions of the magnetic resonance scanning session, subjects lay supine on a narrow bed for the duration of cough threshold testing.

Three milliliters of each dose of capsaicin was loaded into a dedicated nebulizer cup. Subjects were fitted with the modified face mask to which the three nebulizers containing the lowest doses of capsaicin were attached. Subjects were given a verbal cue to prepare for each capsaicin challenge and instructed to take one maximum capacity inhalation on presentation of the stimulus and then to exhale the stimulus without delay. Immediately after each exhalation, subjects rated their urge to cough on a scale from zero (no urge to cough) to 10 (maximum urge to cough). After $2 \mathrm{~min}$, the procedure was repeated with the next highest dose of capsaicin until the concentration that evoked two or more coughs was reached. Subjects were told to breathe through their mouths during each capsaicin challenge.

\section{Brain imaging session}

Image acquisition. Anatomical and functional brain images were collected at the Murdoch Children's Research Institute (Melbourne, Australia) using a Siemens Trio 3T scanner (Siemens) with a 32 channel head coil. Anatomical T1-weighted images were acquired in the sagittal plane [208 slices; $0.90 \mathrm{~mm}$ thickness; $0.94 \times 0.94 \mathrm{~mm}^{2}$ in-plane resolution; echo time (TE), $2.54 \mathrm{~ms}$; repetition time (TR), $1900 \mathrm{~ms}$; flip angle, $9^{\circ}$ ]. Functional brain imaging was performed using the BOLD contrast.

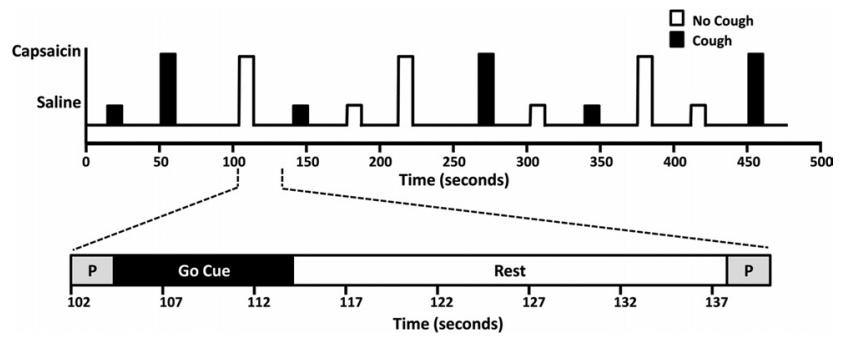

Figure 1. Experimental protocol for fMRI studies. Top schematic, An example of the four functional runs that were performed for each subject, consisting of three presentations of each condition. A classic $2 \times 2$ factorial design (no cough/cough and no capsaicin/capsaicin) was used to assess brain BOLD responses for saline challenge, voluntary cough, capsaicin-evoked cough, suppression of evoked cough, and the interaction between capsaicin challenge and coughing. Bottom schematic, An expanded view of one challenge. A brief visual cue was used to prepare (P) for challenge and to instruct subjects how to respond (i.e., "cough" or "don't cough"), followed by a "Go Cue" during which capsaicin or saline was blindly presented. Challenges were timed so that the nebulized gas reached the subject precisely at the onset of a single maximal inspiratory effort and was then replaced by medical air immediately at the peak of inspiration. Subjects performed the required task and then breathed normally (Rest) until the next preparatory cue. Rest periods were variable in duration between 18 and $36 \mathrm{~s}$.

Echo-planar images (EPIs) were acquired in the transaxial plane (36 slices; $4.5 \mathrm{~mm}$ thickness; $3.28 \times 3.28 \mathrm{~mm}^{2}$ in-plane resolution; TE, 32 ms; TR, $2000 \mathrm{~ms}$; flip angle, $90^{\circ}$ ).

$f M R I$ protocol. Before entering the scanner, all subjects were retested to confirm their C2 threshold. In cases in which the C2 threshold had increased or decreased since the initial psychophysical testing session, the newly determined C2 concentration was used as the capsaicin stimulus in the subsequent fMRI scans. During image acquisition, subjects lay comfortably on the scanner bed with their head stabilized with foam padding. Subjects were provided with hearing protection and were fitted with the face mask and nebulizing apparatus used in the previous session. Subjects were also fitted with a respiratory monitor that provided on-line measurements throughout the experiment. Four fMRI scans of $472 \mathrm{~s}$ duration (236 volumes/scan) were performed during which EPI data were collected under four conditions as follows: (1) exhalation of saline without coughing (no cough, no capsaicin: "saline challenge"), (2) cough after saline inhalation (cough, no capsaicin: "voluntary cough"), (3) cough after capsaicin challenge (cough, capsaicin: "evoked cough"), and (4) exhalation of capsaicin without coughing (no cough, capsaicin: "cough suppression").

Each of the four fMRI scans collected from each participant included 12 event trials ( 3 of each condition) separated by rest periods of $18-36 \mathrm{~s}$ (Fig. 1), resulting in a total of 48 event trials (12 repeats of each condition). Stimuli (capsaicin or saline) were delivered for one deep inhalation in random order. Visual cues were used to coincide the timing of the subjects' inhalation with the onset of each challenge. Subjects were instructed to take one maximal inhalation on the appearance of a "GO" cue. A preparatory cue presented $3 \mathrm{~s}$ before the "GO" cue advised subjects whether to "cough" or "don't cough" after the maximal inhalation. The visual cue also incorporated information outside the subject's field of view to prompt the experimenter for stimulus onset. On-line respiratory monitoring (visible only to the experimenter) was used to coincide the timing of stimulus offset with the beginning of exhalation. After each challenge, the subject's compliance with the protocol (i.e., cough or no cough) was confirmed by visually inspecting the on-line respiratory recording. At the completion of fMRI scanning, subjects were asked to list any additional sensations (other than the urge to cough) that they experienced during the capsaicin challenges. Fifteen of the 19 subjects included in the study reported no additional sensations. Of the four subjects who reported additional sensations, one experienced "watery eyes," one had "a slight urge to sneeze," one could "taste the capsaicin," and one felt a "slight tingling on the tongue."

\section{Data analysis}

Statistical analyses of psychophysical data were performed using SPSS 15.0 for Windows. Means and SDs were calculated for age and capsaicin 
cough thresholds for male and female subjects. Independent $t$ tests were performed to examine sex differences, and dependent $t$ tests were performed to examine session effects on the concentration of capsaicin required to evoke two coughs. Preprocessing and analysis of fMRI data was performed by using FEAT, version 5.98, in FMRIB Software Library (FSL) (www.fmrib.ox.ac.uk/fsl/). Each individual EPI time series was realigned to correct for head motion using a six parameter, rigid-body method. Data were spatially smoothed with a Gaussian kernel of $6 \mathrm{~mm}^{3}$ full width at half-maximum, and nonbrain tissue was removed from the images. To reduce low-frequency noise, a high-pass temporal filter with a cutoff frequency of $0.01 \mathrm{~Hz}$ was applied to the time series data.

General linear modeling analysis of individual time series data was performed with local autocorrelation correction (Woolrich et al., 2001). Regressors convolved with the hemodynamic response function were generated to model each of the events incorporated in the experimental protocol, including the preparatory cue, go cue, saline challenge, voluntary cough, evoked cough, and suppressed cough. The act of coughing was expected to cause a decrease in the mean global signal of the brain because of the combined effects of head motion and periodic local field inhomogeneity (Birn et al., 2008). The timing of cues was designed to prompt coughing events within the acquisition of a single volume. Time series were visually inspected to ensure that coughing-related signal changes were confined to the expected volume by noting interslice intensity differences that typically manifest when movement occurs during an interleaved acquisition. To account for such rapid cough-related signal changes, we designed an analysis model that incorporated an additional regressor, not convolved with the hemodynamic response, which represented the time at which cough occurred as confirmed by visual inspection (see Fig. 2). In addition, the six motion correction parameters were included as regressors of no interest.

Other physiological processes, which include moment-to-moment fluctuations in heart rate, respiration, and arterial $\mathrm{CO}_{2}$ levels, can also confound identification of functional activation or result in additional false-positive BOLD signal changes, particularly when they are correlated with the task of interest (Birn et al., 2008, 2009). In particular, respiration-induced BOLD signal changes typically occur at very low temporal frequencies $(<0.1 \mathrm{~Hz})$ and are not removed by standard correction techniques such as temporal filtering (Birn et al., 2008). Given that even very short periods of breath holding or subtle variations in breathing rate and/or depth can significantly influence BOLD signal changes, we adopted the method described by Birn et al. (2008) in which three additional nuisance regressors were computed from the time series data and included in the model to reduce the influence of respiratoryrelated physiological noise. The nuisance regressors were (1) the mean global signal time series from nonactivated regions of the brain acquired through an iterative process first described for positron emission tomography data by Andersson (1997), (2) a CSF regressor, computed by averaging the BOLD signal time series from the lateral ventricle, and (3) the time series from a voxel in the brain with the highest SD. Inclusion of these three nuisance regressors in the general linear statistical model has been previously shown to significantly reduce the influence of respiratory changes on the identification of task-related neuronal activation (Lund et al., 2006; Birn et al., 2009).

Contrasts were performed to identify brain activation during each of the four experimental contingencies: saline challenge (no cough, no capsaicin), voluntary cough (cough, no capsaicin), evoked cough (cough, capsaicin), and cough suppression (no cough, capsaicin). The latter three events were then contrasted with saline challenge to reveal activation patterns unique to the three events of interest. As a second level of analysis, brain activation during coughing was identified by contrasting the two events involving cough with the no-cough events, represented mathematically as [cough, no capsaicin + cough, capsaicin] $>$ [no cough, no capsaicin + no cough, capsaicin]. Similarly, activations associated with capsaicin challenge without the confound of coughing were identified (i.e., [cough, capsaicin + no cough, capsaicin] $>$ [ cough, no capsaicin + no cough, no capsaicin]). Finally, the positive and negative interactions between coughing and capsaicin were assessed. Brain regions that showed a capsaicin and cough positive interaction were identified as regions where the BOLD signal increase after evoked cough compared
Table 1. Subject characteristics and capsaicin cough thresholds

\begin{tabular}{cllcc}
\hline ID no. ${ }^{a}$ & Sex & $\begin{array}{l}\text { Age } \\
\text { (years) }\end{array}$ & $\begin{array}{l}\text { Session 1C2 } \\
(\mu \mathrm{m})\end{array}$ & $\begin{array}{l}\text { Session 2 C2 } \\
(\mu \mathrm{m})\end{array}$ \\
\hline 1 & Male & 35 & 15.63 & 15.63 \\
2 & Female & 36 & 7.81 & 7.81 \\
3 & Male & 49 & 15.63 & 15.63 \\
4 & Female & 21 & 3.91 & 3.91 \\
5 & Female & 21 & 3.91 & 3.91 \\
6 & Female & 22 & 1.95 & 1.95 \\
9 & Male & 21 & 15.63 & 15.63 \\
10 & Male & 24 & 3.91 & 3.91 \\
11 & Male & 21 & 3.91 & 3.91 \\
12 & Male & 23 & 31.25 & 31.25 \\
13 & Male & 28 & 7.81 & 3.91 \\
14 & Female & 27 & 1.95 & 0.25 \\
15 & Female & 19 & 0.98 & 0.98 \\
16 & Male & 20 & 15.63 & 31.25 \\
17 & Male & 18 & 7.81 & 7.81 \\
18 & Male & 20 & 1.95 & 3.91 \\
19 & Male & 19 & 7.81 & 7.81 \\
20 & Female & 33 & 7.81 & 3.91 \\
21 & Male & 26 & 7.81 & 7.81 \\
Mean & Male & $25.6 \pm 6.2$ & $8.71 \pm 1.26^{b}$ & $9.33 \pm 1.31^{b}$ \\
Mean & Female & $24.5 \pm 11.2$ & $3.23 \pm 1.35^{b}$ & $2.29 \pm 1.41^{b}$ \\
\hline
\end{tabular}

${ }^{a}$ Data from two subjects were excluded from all analyses because of excessive event-related head motion (no.7) and a failure to comply with the experimental protocol (no. 8).

${ }^{b} \mathrm{C} 2$ (concentration of capsaicin required to evoke two coughs) shown as geometric mean $\pm \mathrm{SE}$.

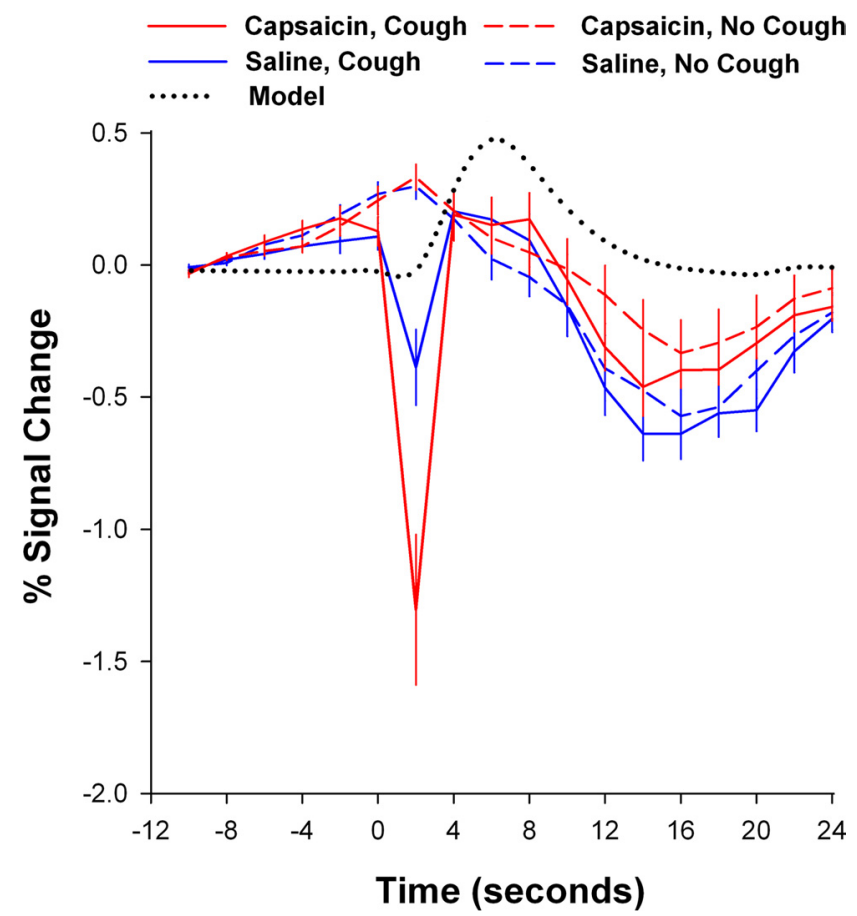

Figure 2. Mean time courses of the global BOLD signal changes associated with capsaicin and cough events relative to the analytical model used.

with saline challenge was significantly greater than the BOLD signal increase after voluntary coughing compared with cough suppression (i.e., $[$ cough, capsaicin + no cough, no capsaicin] $>$ [cough, no capsaicin + no cough, capsaicin]). The negative interaction identified brain regions where the BOLD signal increase after voluntary coughing compared with cough suppression was significantly greater than the BOLD signal increase after capsaicin evoked cough compared with saline challenge (i.e., $[$ cough, no capsaicin + no cough, capsaicin $]>[$ cough, capsaicin + no cough, no capsaicin]). 
Table 2. Regions showing increased activation associated with saline exhalation, voluntary cough, evoked cough, and suppressed cough

\begin{tabular}{|c|c|c|c|c|c|c|c|c|c|c|c|c|c|c|c|c|}
\hline \multirow[b]{3}{*}{ Region } & \multicolumn{4}{|c|}{ Saline challenge } & \multicolumn{4}{|c|}{ Voluntary cough } & \multicolumn{4}{|c|}{ Evoked cough } & \multicolumn{4}{|c|}{ Suppressed cough } \\
\hline & \multicolumn{3}{|c|}{ Peak voxel coordinate } & \multirow[b]{2}{*}{ Zscore } & \multicolumn{3}{|c|}{ Peak voxel coordinate } & \multirow[b]{2}{*}{ Zscore } & \multicolumn{3}{|c|}{ Peak voxel coordinate } & \multirow[b]{2}{*}{ Zscore } & \multicolumn{3}{|c|}{ Peak voxel coordinate } & \multirow[b]{2}{*}{ Zscore } \\
\hline & $x$ & $y$ & $z$ & & $x$ & $y$ & $z$ & & $x$ & $y$ & $z$ & & $x$ & $y$ & $z$ & \\
\hline \multirow[t]{6}{*}{ Cingulate cortex } & & & & & & & & & & & & & 0 & 44 & 2 & 3.22 \\
\hline & & & & & 4 & 38 & 4 & 3.04 & & & & & 4 & 42 & 2 & 4.39 \\
\hline & 4 & 24 & 28 & 3.62 & 0 & -2 & 38 & 4.49 & 6 & 22 & 22 & 4.53 & 2 & 26 & 28 & 5.29 \\
\hline & -2 & 20 & 32 & 2.94 & 2 & 2 & 38 & 4.35 & -2 & 20 & 22 & 4.56 & -2 & 26 & 22 & 4.97 \\
\hline & & & & & 0 & -16 & 40 & 3.58 & 6 & -22 & 32 & 3.22 & 4 & -20 & 30 & 3.78 \\
\hline & & & & & -2 & -14 & 40 & 3.97 & -4 & -16 & 30 & 4.14 & -2 & -22 & 30 & 3.66 \\
\hline Superior frontal gyrus & 6 & 12 & 58 & 3.91 & & & & & -2 & 26 & 22 & 5.29 & & & & \\
\hline Middle frontal gyrus & 44 & 12 & 50 & 4.04 & & & & & & & & & & & & \\
\hline & 40 & 28 & 34 & 3.79 & 36 & 56 & 12 & 4.05 & & & & & 44 & 22 & 44 & 3.40 \\
\hline & -34 & 44 & 24 & 4.05 & -32 & 48 & 22 & 3.59 & & & & & -36 & 28 & 34 & 3.34 \\
\hline Pre-SMA & & & & & & & & & & & & & 6 & 34 & 42 & 3.50 \\
\hline & & & & & & & & & & & & & -2 & 26 & 44 & 2.86 \\
\hline SMA & 4 & -10 & 58 & 3.01 & 4 & 2 & 62 & 4.88 & 6 & -10 & 60 & 3.09 & 4 & 8 & 52 & 3.37 \\
\hline & -6 & 8 & 60 & 2.79 & -2 & 2 & 60 & 4.07 & -8 & -10 & 68 & 3.36 & 0 & 2 & 52 & 2.62 \\
\hline Premotor cortex & 54 & 2 & 42 & 3.54 & & & & & & & & & 56 & 2 & 40 & 5.16 \\
\hline Sensorimotor cortex & & & & & 26 & -28 & 62 & 4.02 & 22 & -30 & 60 & 4.13 & & & & \\
\hline & & & & & -22 & -34 & 64 & 4.27 & -20 & -34 & 60 & 4.03 & & & & \\
\hline & & & & & 50 & -8 & 54 & 3.20 & 56 & -2 & 40 & 4.62 & & & & \\
\hline & & & & & -50 & -8 & 46 & 3.68 & & & & & & & & \\
\hline & & & & & 46 & -10 & 34 & 3.83 & 36 & -14 & 40 & 4.00 & & & & \\
\hline & & & & & -46 & -10 & 40 & 4.64 & -46 & -12 & 42 & 3.86 & -46 & -12 & 40 & 3.14 \\
\hline & & & & & 44 & -12 & 30 & 4.27 & 44 & -8 & 30 & 4.57 & 46 & -8 & 34 & 2.78 \\
\hline & & & & & -42 & -10 & 30 & 4.80 & -64 & -20 & 32 & 5.06 & -40 & -18 & 32 & 3.38 \\
\hline Operculum & 58 & -14 & 14 & 3.68 & 64 & -4 & 10 & 5.22 & -60 & 4 & 10 & 5.15 & -56 & -2 & 10 & 5.18 \\
\hline & -56 & -4 & 12 & 3.11 & -46 & -12 & 18 & 4.67 & 64 & -4 & 10 & 5.12 & 58 & -4 & 8 & 5.48 \\
\hline Posterior cingulate cortex & & & & & & & & & 8 & -32 & 42 & 3.41 & 6 & -26 & 36 & 3.49 \\
\hline & & & & & & & & & -14 & -38 & 42 & 4.28 & -2 & -22 & 30 & 3.66 \\
\hline Inferior parietal lobule & 64 & -42 & 26 & 4.53 & 64 & -34 & 24 & 4.17 & 62 & -36 & 26 & 4.69 & 60 & -42 & 42 & 4.70 \\
\hline & -60 & -42 & 40 & 4.44 & -62 & -36 & 30 & 3.51 & -64 & -38 & 34 & 3.87 & -58 & -40 & 46 & 4.36 \\
\hline Middle temporal gyrus & 56 & -52 & -2 & 3.10 & & & & & & & & & & & & \\
\hline & -50 & -62 & 0 & 4.06 & & & & & & & & & & & & \\
\hline Anterior insula & 38 & 22 & 4 & 2.86 & & & & & 34 & 14 & 4 & 3.40 & 36 & 20 & 2 & 3.51 \\
\hline & & & & & & & & & -32 & 10 & 6 & 3.41 & -34 & 16 & 2 & 3.47 \\
\hline Mid-insula & & & & & 44 & 4 & 0 & 3.13 & 36 & -12 & 8 & 3.12 & 46 & 12 & -4 & 3.85 \\
\hline & -40 & 10 & 2 & 2.81 & -44 & 2 & -2 & 4.39 & -40 & -12 & 8 & 4.46 & -38 & 10 & 4 & 3.84 \\
\hline Posterior insula & & & & & 40 & -8 & 8 & 2.98 & 34 & -22 & 8 & 3.33 & & & & \\
\hline & & & & & -38 & -12 & 8 & 4.33 & -32 & -26 & 10 & 3.69 & & & & \\
\hline Caudate & & & & & & & & & & & & & 16 & 22 & 2 & 3.57 \\
\hline Lentiform nuclei & & & & & 16 & 10 & -6 & 3.01 & & & & & 16 & 12 & -6 & 3.06 \\
\hline Thalamus & & & & & & & & & 10 & -18 & 4 & 2.99 & 12 & -6 & 10 & 2.84 \\
\hline & & & & & -8 & -2 & 0 & 3.62 & -14 & -18 & 4 & 3.48 & -10 & -6 & 10 & 3.25 \\
\hline Midbrain & & & & & 6 & -32 & -12 & 3.54 & 4 & -32 & -14 & 3.84 & & & & \\
\hline & & & & & -10 & -24 & -14 & 4.27 & -8 & -26 & -14 & 4.37 & & & & \\
\hline Pons & & & & & & & & & 8 & -32 & -28 & 3.17 & & & & \\
\hline & & & & & & & & & -8 & -36 & -28 & 3.86 & & & & \\
\hline Rostral medulla & & & & & & & & & 0 & -38 & -52 & 2.52 & & & & \\
\hline & & & & & & & & & -4 & -38 & -50 & 3.40 & & & & \\
\hline Caudal medulla & & & & & & & & & 0 & -40 & -66 & 2.86 & & & & \\
\hline & & & & & & & & & -4 & -44 & -64 & 3.26 & & & & \\
\hline Cerebellum & & & & & 20 & -70 & -22 & 4.66 & 4 & -46 & -24 & 5.02 & & & & \\
\hline & & & & & -24 & -64 & -26 & 4.54 & -8 & -62 & -20 & 4.58 & & & & \\
\hline
\end{tabular}

The coordinates of maximally activated voxels are given in MNI space where $x, y$, and $z$ coordinates represent the medial-lateral, anterior-posterior, and superior-inferior distances (in millimeters) from the anterior commissure. SMA, Supplementary motor area.

All activations identified at cluster-level significance of $p<0.05$ (corrected).

For higher-level (group) analysis, individual subject's data were normalized to standard stereotactic space, as defined by the Montreal Neurological Institute (MNI) 152 brain template. Each subject's mean EPI image was first coregistered with their high-resolution T1 images, which were in turn spatially normalized to the standard MNI template. The transformation parameters were then applied to the subject's statistical parametric image to enable group averaging. Group effects were identified with a mixed model that incorporated variance within session and across time (fixed effects) and cross-session variance (random effects). The group statistical parametric maps [ $Z$ (Gaussianized $T / F$ ) statistics] were thresholded to include voxels with values of $Z>2.3$ and that formed contiguous groupings with a cluster probability threshold of $p<$ 0.05 , corrected for multiple comparisons [based on Gaussian random field theory (Worsley et al., 1992)].

Time courses were extracted to identify hemodynamic response properties in the regions of interest (ROIs) showing either a positive or neg- 


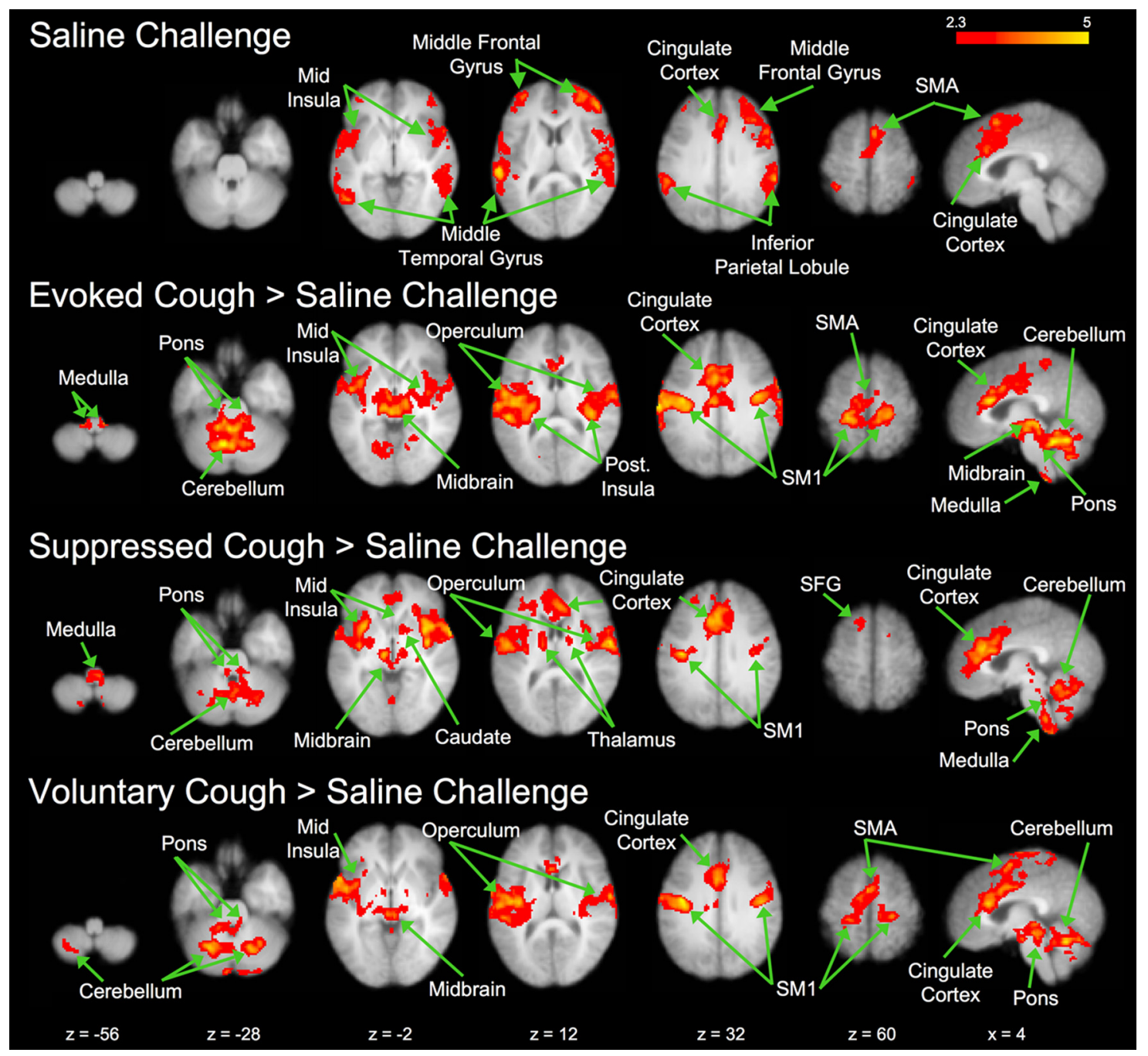

Figure 3. Representative BOLD signal responses associated with saline challenge and evoked cough, suppressed cough or voluntary cough after contrasting activations with saline challenge. The left side of each map corresponds to the left side of the brain. SM1, Primary sensorimotor cortex; SMA, supplementary motor area; SFG, superior frontal gyrus. See Tables 2 and 3 for a list of activated regions.

ative interaction between cough and capsaicin. Each ROI volume was composed of the significantly activated voxels situated within the selected anatomical region, as defined by the Harvard-Oxford Cortical and Subcortical Structural Atlases implemented in FSLView, version 3.0 (www. fmrib.ox.ac.uk/fsl/fslview). Using the FEAT query tool in FSLView, mean percentage signal changes were extracted from the ROIs for each of the four experimental conditions.

\section{Results}

Capsaicin-evoked cough thresholds

Inhalations of escalating concentrations of capsaicin elicited progressively stronger ratings of a perceived urge to cough and, eventually, resulted in spontaneous coughing. Consistent with previous reports (Dicpinigaitis and Rauf, 1998; Mazzone et al., 2007), the concentration of capsaicin required to elicit two spontaneous coughs was lower in female subjects compared with males during both session $1\left(t_{(17)}=2.74 ; p<0.05\right)$ and session 2 $\left(t_{(17)}=3.28 ; p<0.01\right)$ (Table 1). However, the average capsaicin concentration thresholds required to evoke two coughs (C2) did not differ significantly across the two experimental sessions for either males $\left(t_{(11)}=-0.56\right.$; n.s $)$ or females $\left(t_{(6)}=1.34\right.$; n.s $)$.
Effect of coughing on BOLD signal measurements

The global BOLD signal was adversely affected at the exact moment of coughing in all subjects (Fig. 2). This decrease in signal intensity was significantly greater during evoked cough compared with voluntary cough $\left(-1.30\right.$ vs $-0.33 \%$, respectively; $t_{(18)}=$ $-4.817, p<0.001)$. Furthermore, the changes in the global signal were highly correlated $\left(r^{2}=0.64-0.74\right)$ with head movement, and the latter was also significantly greater during evoked cough compared with voluntary cough $\left(0.42\right.$ vs $0.30 \mathrm{~mm} ; t_{(18)}=4.18$, $p<0.001)$. These observations likely reflect the more "uncontrollable" nature of reflex evoked cough compared with voluntary cough. Nevertheless, the cough-related BOLD signal loss recovered within one TR for both evoked cough and voluntary cough, and the mean global signal of the brain had returned to precough baseline levels before the modeled peak of the hemodynamic response for each cough event (Fig. 2).

Regional brain activity associated with saline challenge, voluntary cough, evoked cough, and cough suppression As a first-level analysis, we assessed regions showing significant BOLD signal response during each of the four conditions (saline 
Table 3. Regions showing increased activation associated with the contrasts of voluntary cough $>$ saline challenge, evoked cough $>$ saline challenge, and suppressed cough $>$ saline challenge

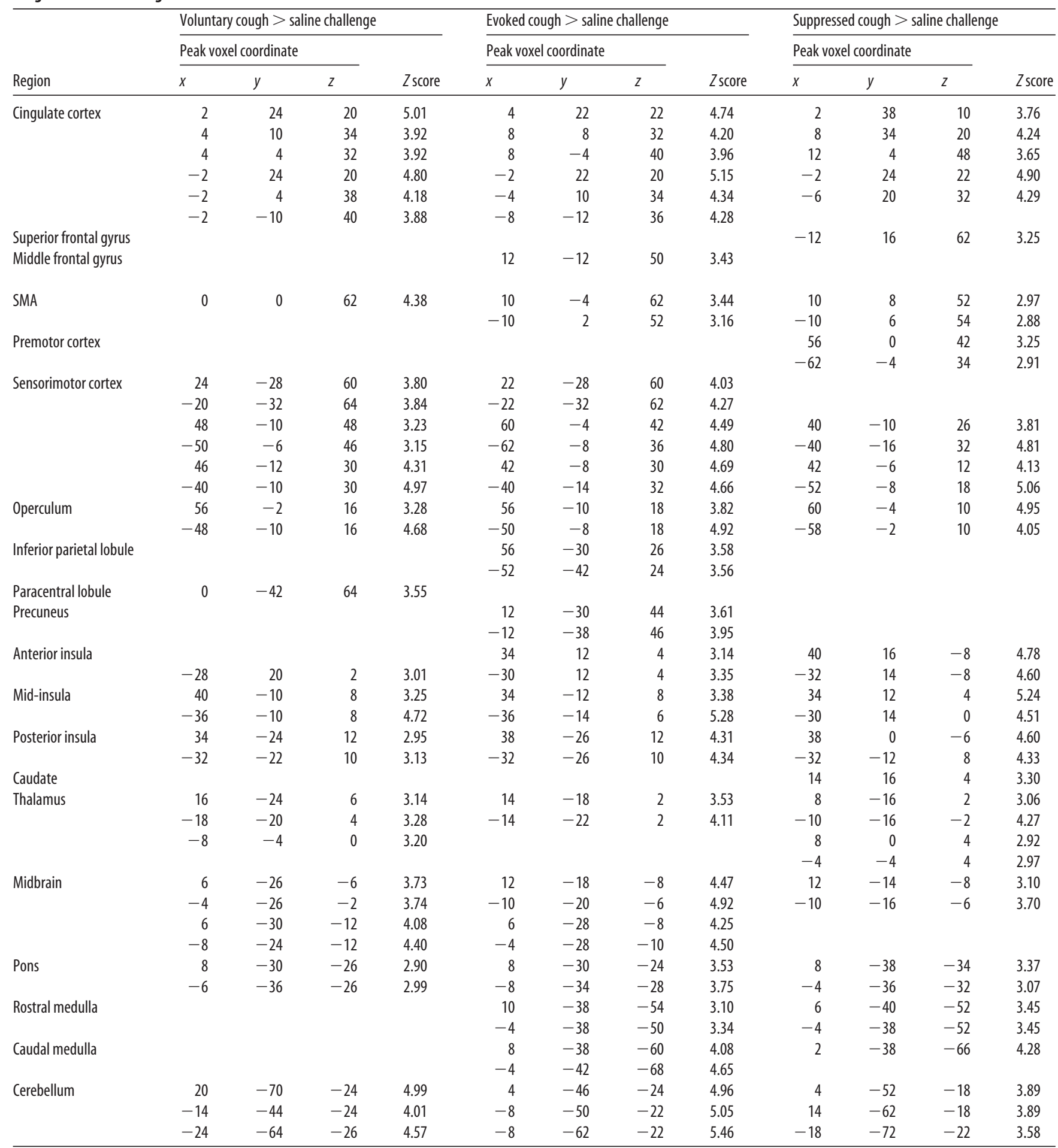

The coordinates of maximally activated voxels are given in MNI space where $x, y$, and $z$ coordinates represent the medial-lateral, anterior-posterior, and superior-inferior distances (in millimeters) from the anterior commissure. SMA, Supplementary motor area.

All activations identified at cluster-level significance of $p<0.05$ (corrected).

challenge, voluntary cough, capsaicin-evoked cough, and cough suppression) (Table 2). Each condition (including saline challenge) was associated with increased activation in the supplementary motor area, operculum, and anterior mid-cingulate cortex. Brain regions commonly activated by evoked cough, voluntary cough, and cough suppression included the primary motor and somatosensory cortices, and posterior mid-cingulate cortex.
Unique activation associated with cough suppression was seen in the pre-supplementary motor area and caudate. In addition, activation clusters in the rostral and caudal medulla were identified for evoked cough but were not observed during voluntary cough, suppressed cough, or saline challenge (Table 2). Additional contrasts were made using three of the four conditions to identify BOLD signal increases compared with the saline challenge (Fig. 3, 


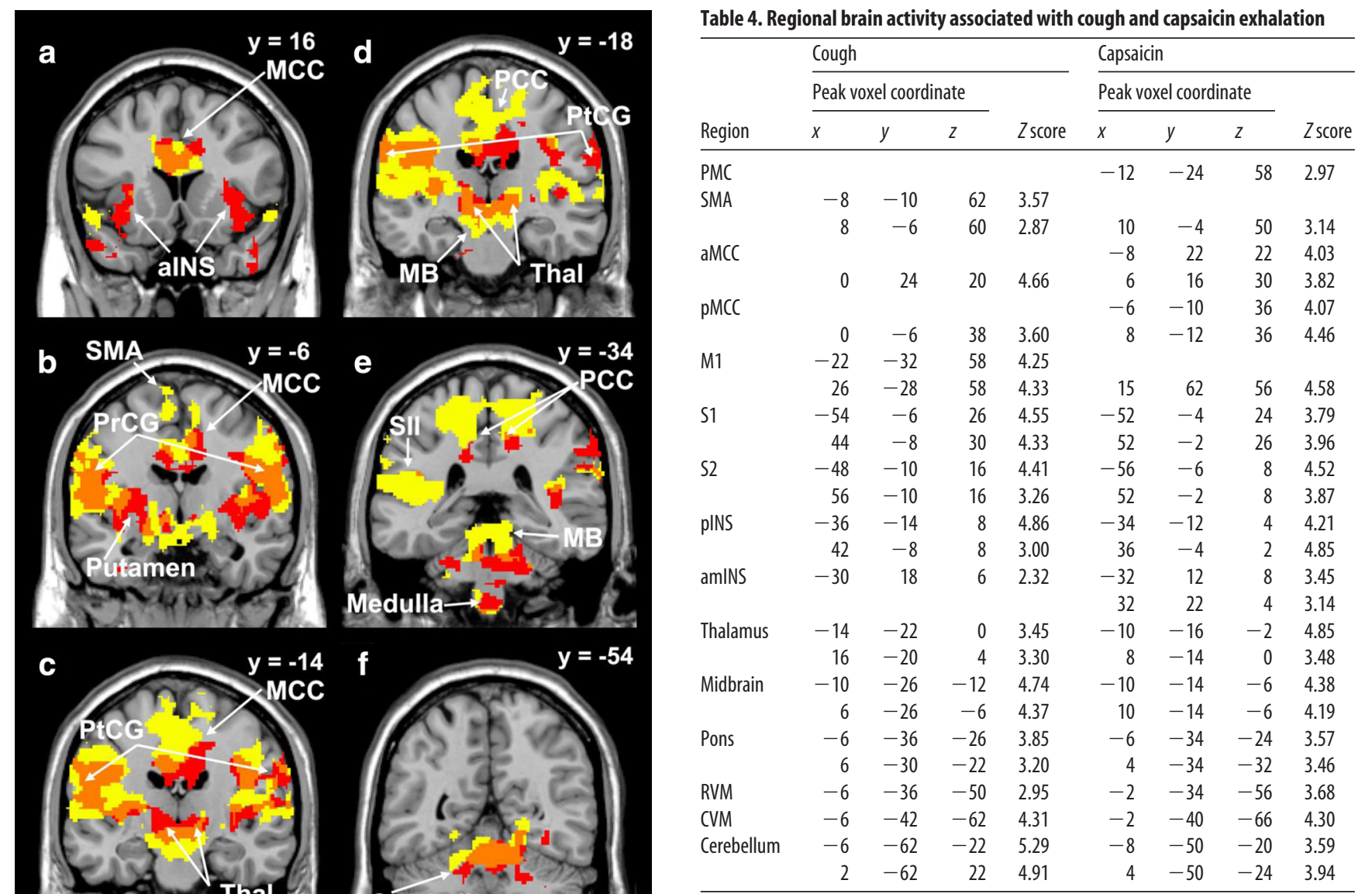

The main effect for cough was determined by contrasting coughing events with noncoughing events (evoked cough + voluntary cough $>$ suppressed cough + saline challenge). The main effect for capsaicin was determined by contrasting capsaicin events with saline events (evoked cough + suppressed cough $>$ voluntary cough + saline challenge). The coordinates of maximally activated voxels are given in MNI space where $x, y$, and $z$ coordinates represent the medial-lateral, anterior-posterior, and superior-inferior distances (in millimeters) from the anterior commissure. PMC, Premotor cortex; SMA, supplementary motor area; aMCC, anterior mid-cingulate cortex; pMCC, posterior mid-cingulate cortex; $\mathrm{M} 1$, primary motor cortex; $\mathrm{S1}$, primary somatosensory cortex; $\mathrm{S2}$, secondary somatosensory cortex; pINS, posterior insula cortex; amINS, anterior/mid-insula cortex; RVM, rostral ventral medulla; CVM, caudal ventral medulla.

All activations identified at cluster-level significance of $p<0.05$ (corrected)

responses associated with capsaicin challenge only (red), cough only (yellow), or both capsaicin and cough (orange). The left side of each map corresponds to the left side of the brain. MCC, Mid-cingulate cortex; aINS, anterior insula cortex; PCC, posterior cingulate cortex; PtCG, postcentral gyrus; MB, midbrain; Thal, thalamus; SMA, supplementary motor area; PrCG, precentral gyrus; SII, secondary somatosensory cortex. See Table 4 for a list of activated regions.

Table 3). For evoked cough, the contrast with saline challenge showed activation in the left cerebellum that was not identified for evoked cough alone. Several regions that showed activation for voluntary cough and cough suppression were not observed when the conditions were individually compared with saline challenge, including the left and right inferior parietal lobules and the middle frontal gyri in both hemispheres. The contrast between cough suppression and saline challenge showed activations in the cerebellar lobes, medulla, and midbrain that were not observed for the cough suppression activation alone. Pontine activation was observed when voluntary cough was compared with saline challenge but was not observed for voluntary cough alone.

Regional brain activity associated with cough and capsaicin The data generated by the first level of analysis highlights regional activations associated with each experimental condition but provides minimal insights in the role of each activated brain region in the regulation of coughing. As a second level of analysis, we compared BOLD signal responses that differentiated the sensory and motor components of cough by contrasting brain activity during coughing with brain activity during stimulus exhalation without cough, regardless of the nature of the initial stimulus. We observed that coughing was associated with a widely distributed network of brain activity, which included activation in the midcingulate cortex extending to the supplementary motor area, and bilateral activity in the primary somatosensory and motor cortices, secondary somatosensory cortex, insula, thalamus, midbrain, brainstem, and cerebellum (Fig. 4, Table 4). Conversely, the unique BOLD attributes of the capsaicin stimulus, independent of cough, were identified by contrasting brain activity resulting from the presence of capsaicin compared with its absence. Activations were observed in the supplementary motor area, primary and secondary somatosensory cortices, anterior and midcingulate cortices, insula, thalamus, midbrain, brainstem, and cerebellum (Fig. 4, Table 4).

Brain regions showing an interaction between capsaicin and cough

The BOLD signal attributes of an evoked cough may be related to brain activations that are not simply predicted by capsaicin or cough alone. Consistent with this, positive interactions between capsaicin and cough were noted in the primary motor and somatosensory cortices, posterior cingulate and posterior midcingulate cortex (pMCC), the posterior insula, and the premotor cortex (Fig. 5, Table 5). Conversely, negative interaction effects 

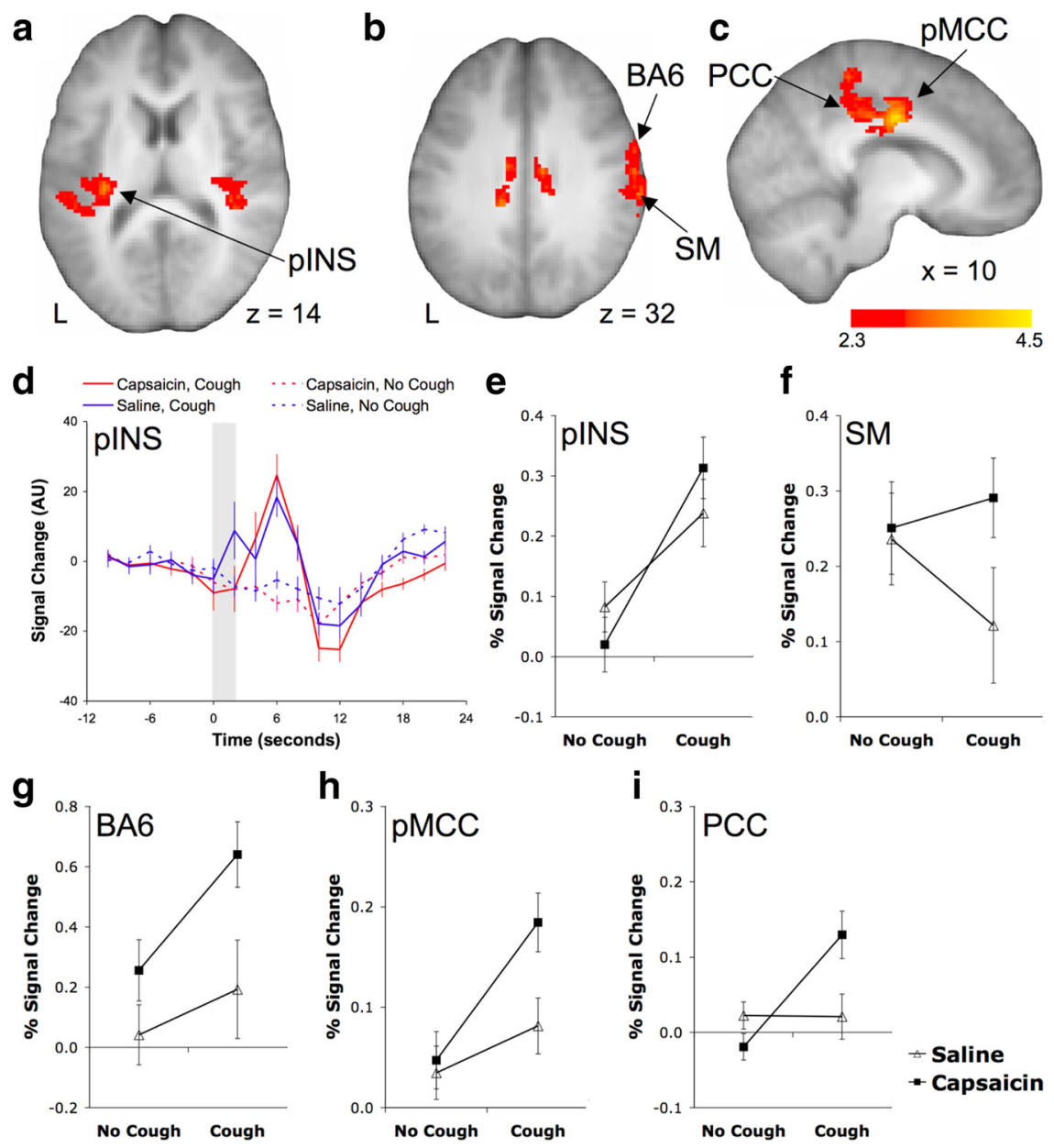

Figure 5. Brain regions showing a positive interaction between cough and capsaicin for the following: the posterior insula (pINS) (a), the primary sensory/ motor cortex (SM1) and premotor cortex (BA6) (b), and the posterior mid-cingulate (pMCC) and posterior cingulate $(\mathrm{PCC})$ cortices $(\boldsymbol{c})$. $\boldsymbol{d}$, The BOLD signal time course in the pINS during each of the four experimental conditions. The shaded region represents the timing of cough events. $\boldsymbol{e}-\boldsymbol{i}$, Mean ( \pm SE) percentage BOLD signal changes for each of the conditions for the pINS $(\boldsymbol{e})$, SM1 $(\boldsymbol{f}), \operatorname{BA6}(\boldsymbol{g})$, pMCC $(\boldsymbol{h})$, and PCC (i). See Table 5 for a list of activated regions. cued maximal inhalation of capsaicin or saline. Studies from other laboratories have directly assessed the neural correlates of volitional breathing control. Notably, volitional inspirations evoke discrete activations in the superior motor cortex in an area thought to represent diaphragmatic movements (Ramsay et al., 1993; Evans et al., 1999), whereas volitional expirations (in some paradigms only) also evoke motor cortex activations, but typically in regions inferior to those associated with inspiration (Ramsay et al., 1993; Evans et al., 1999). Interestingly, we did not observe any activation in the primary motor cortex associated with saline challenge without cough, perhaps as we modeled the expiratory phase, which was typically passive for this respiratory event. In other studies, breath holding activates the dorsolateral prefrontal cortex, premotor cortex, mid-insula, anterior mid-cingulate cortex, inferior frontal gyrus, and middle temporal gyrus (McKay et al., 2008). Furthermore, volitional hyperpnea, volitional inspirations, and volitional sniffing are accompanied by signal changes in the prefrontal cortex, supplementary motor area, superior frontal gyrus, middle frontal gyrus, striatum, and cerebellum (Evans et al., 1999; McKay et al., 2003, 2008; Simonyan et al., 2007; Koritnik et al., 2009). Consistent with this, we noted responses in the same regions after saline challenges without coughing highlighting commonalities in the neural substrates controlling a variety of volitional respiratory activities. were observed in the supplementary motor area, anterior insula, anterior mid-cingulate cortex, inferior frontal gyri, and the prefrontal cortex (Fig. 6, Table 5).

\section{Discussion}

To address the hypothesis that reflex and voluntary control of coughing include supramedullary brain mechanisms, we compared BOLD responses associated with saline challenge followed by normal breathing, voluntary cough, capsaicin-evoked cough, and cough suppression after capsaicin challenge. Thus, we compared and contrasted experimental conditions that represented (1) neither a cough sensory stimulus nor a cough motor event (saline challenge), (2) no cough sensory stimulus but a cough motor event (voluntary cough), (3) both a cough sensory stimulus and a cough motor event (capsaicin evoked cough), and (4) a cough sensory stimulus without the accompanying cough motor event (cough suppression). Our data indicate the existence of distinct higher brain circuitry for facilitating and suppressing the cough reflex and have therefore provided novel insights into the supramedullary control of cough in humans.

\section{Volitional control of breathing: activations associated with saline challenge}

The successful completion of expiratory events required participants to briefly pause their normal respiratory pattern before a

\section{Supramedullary control of voluntary cough}

Cough can be voluntarily initiated and this may contribute to behavioral coughing in respiratory diseases. A recent functional imaging study provided insights into the supramedullary mechanisms that likely contribute to generating voluntary coughing (Simonyan et al., 2007). The BOLD signal changes that accompanied a voluntary cough were widespread and included activations in the primary motor and sensory cortices, inferior frontal gyrus, operculum, insula, anterior and mid-cingulate cortex, ventral and dorsomedial thalamus, caudate nucleus, putamen, and cerebellum. We used respiratory response modeling to eliminate the confounding effects on the BOLD signal of the brief respiratory modifications that were necessary for the successful completion of cued voluntary coughs and then contrasted voluntary cough activations with those obtained after saline challenge without cough. Our results reveal a similar (albeit slightly more restricted) pattern of brain activation associated with voluntary cough compared with that reported by Simonyan et al. (2007).

\section{Supramedullary activations after capsaicin challenge}

In a previous study, we used capsaicin inhalation to provide insights into the supramedullary circuitry involved in sensing airway irritations (Mazzone et al., 2007). Capsaicin activates TRPV1 (transient receptor potential vanilloid 1) receptors expressed by a 
Table 5. Brain regions showing an interaction effect between capsaicin and coughing

\begin{tabular}{|c|c|c|c|c|c|c|c|c|c|}
\hline \multirow[b]{3}{*}{ Region } & \multicolumn{4}{|c|}{ Positive interaction } & \multirow[b]{3}{*}{ Region } & \multicolumn{4}{|c|}{ Negative interaction } \\
\hline & \multicolumn{3}{|c|}{ Peak voxel coordinate } & \multirow[b]{2}{*}{ Zscore } & & \multicolumn{3}{|c|}{ Peak voxel coordinate } & \multirow[b]{2}{*}{ Zscore } \\
\hline & $x$ & $y$ & $z$ & & & $x$ & $y$ & $z$ & \\
\hline \multirow[t]{3}{*}{ PMC } & -18 & -26 & 62 & 3.12 & PFC & 26 & 50 & 2 & 3.91 \\
\hline & 22 & -22 & 62 & 3.21 & $\mathrm{aMCC}$ & -2 & 24 & 32 & 3.05 \\
\hline & 60 & -2 & 38 & 3.22 & & 2 & 26 & 32 & 3.39 \\
\hline \multirow[t]{2}{*}{ M1 } & -18 & -26 & 58 & 3.54 & SMA & 0 & 0 & 66 & 3.97 \\
\hline & 6 & -36 & 58 & 3.48 & SFG & 4 & 26 & 56 & 3.78 \\
\hline SM & 62 & -12 & 30 & 3.47 & alNS & 42 & 16 & 0 & 3.18 \\
\hline \multirow[t]{2}{*}{ S2 } & -44 & -32 & 22 & 3.26 & IFG & 42 & 28 & -4 & 3.26 \\
\hline & 48 & -26 & 24 & 3.04 & & & & & \\
\hline \multirow[t]{2}{*}{ pINS } & -32 & -26 & 14 & 3.58 & & & & & \\
\hline & 38 & -32 & 14 & 3.22 & & & & & \\
\hline \multirow[t]{2}{*}{ pMCC } & -8 & -12 & 36 & 4.03 & & & & & \\
\hline & 8 & -10 & 38 & 4.15 & & & & & \\
\hline \multirow[t]{2}{*}{$\mathrm{PCC}$} & -8 & -32 & 42 & 3.87 & & & & & \\
\hline & 12 & -26 & 38 & 3.56 & & & & & \\
\hline
\end{tabular}

The coordinates of maximally activated voxels are given in MNI space where $x, y$, and $z$ coordinates represent the medial-lateral, anterior-posterior, and superior-inferior distances (in millimeters) from the anterior commissure. SMA, Supplementary motor area; PMC, premotor cortex; M1, primary motor cortex; SFG, superior frontal gyrus; PFC, prefrontal cortex; aMCC, anterior mid cingulate cortex; $\mathrm{PMCC}$, posterior mid-cingulate cortex; $S M$, primary somatosensory/motor cortex; $\$ 2$, secondary somatosensory cortex; alNS, anterior insula; pINS, posterior insula; PCC, posterior cingulate cortex; IFG, inferior frontal gyrus.

All activations identified at cluster-level significance of $p<0.05$ (corrected).

subset of airway sensory nerves (Materazzi et al., 2009) and is commonly used to study cough in animals and humans and to assess cough reflex sensitivity in patients with airways diseases. The utility of this stimulus is that, when inhaled, capsaicin evokes both coughing and the associated urge to cough (Davenport et al., 2002; Mazzone et al., 2007). Furthermore, capsaicin-evoked coughing can be consciously suppressed (Hutchings et al., 1993; Mazzone et al., 2007). Our present data extend our previous fMRI analysis of capsaicin-evoked urge to cough (Mazzone et al., 2007, 2009b), confirming activations associated with capsaicin challenge that encompass sensory, motor, and limbic structures, some of which overlap with those activated during cough. Additionally, capsaicin challenge includes activations associated with the premotor cortex, supplementary motor area anterior/midinsula that were not evident after coughing.

\section{Supramedullary activations associated with evoked cough and cough suppression}

Theoretical models have predicted that higher brain mechanisms contribute to the control of evoked cough through facilitating or inhibiting of brainstem reflex pathways (Davenport et al., 2002; Bolser et al., 2006; Eccles, 2009). Such models predict interactions between the input arising from the sensory apparatus in the airways and the supramedullary cough motor circuitry, thereby providing the framework for this higher order control. However, despite suggestions that such circuitry exists, there have been no attempts to experimentally validate these models or to describe the neural circuitry involved. The interaction analysis of our factorial experimental design demonstrates the unique features of cough and its suppression, and provides the first insights into the components that comprise this higher level network. The positive interaction revealed brain region activations that describe features of evoked cough, whereas the negative interaction identified relatively higher responses during voluntary cough and cough suppression. These activations can be interpreted with data from previous functional studies to suggest a role for brain regions in supramedullary cough control pathways.
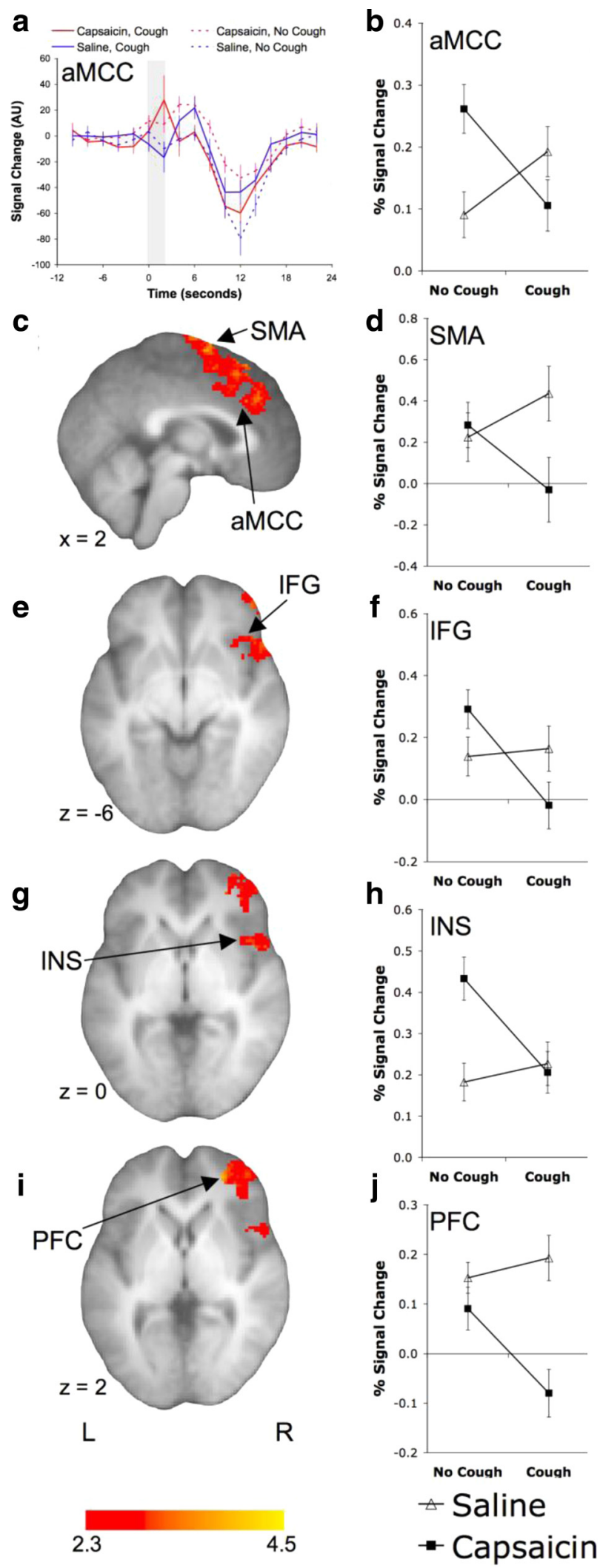

Figure 6. Brain regions showing a negative interaction between cough and capsaicin. The anterior mid-cingulate cortex (aMCC) showing the BOLD signal time course $(\boldsymbol{a})$ and the mean $( \pm$ SE) percentage BOLD signal change $(\boldsymbol{b})$ during each of the four conditions. The shaded region of $\boldsymbol{a}$ represents the timing of cough events. The statistical parametric maps and percentage BOLD signal change for the supplementary motor area (SMA) $(\boldsymbol{c}, \boldsymbol{d})$, inferior frontal gyrus $(\mathrm{IFG})(\boldsymbol{e}, \boldsymbol{f})$, mid/anterior insula (INS) $(\boldsymbol{g}, \boldsymbol{h})$, and prefrontal cortex (PFC) (i,j). See Table 5 for a list of activated regions. 
Activations in the posterior insula are associated with evoked and (to a lesser extent) voluntary cough, but not cough suppression. Stimulation of the posterior insula in monkeys evokes contralateral muscle contractions, indicative of a contribution to motor control (Sugar et al., 1948; Shima et al., 1991). The posterior insula may contribute to the generation of cough or the control of accessory muscles associated with coughing (e.g., oral structures, trunk muscles, etc.) but not cough suppression. A comparable pattern of activation is replicated in the primary motor cortex (BA4) and paracentral lobule, as well as in the posterior mid-cingulate cortex, all of which have roles in motor control. For example, pMCC activations accompany voluntary movements in monkeys and humans, and stimulation of the posterior cingulate cortex evokes short-latency skeletal muscle responses that may be involved in the orientation of the body to nociceptive stimuli (Vogt, 2005). The premotor cortex (BA6) is involved in orolaryngeal movements and is activated by coughing. However, this region additionally shows strong activations during cough suppression, perhaps suggestive of a more generalized role in the control of laryngeal motor tasks. By contrast, activations in the posterior cingulate cortex region are limited to capsaicin-evoked coughing. The posterior cingulate is an anatomically and functionally distinct region of the cingulate cortex (Vogt, 2005). It receives afferent input from thalamic nuclei and has been shown to have functional (during voluntary voice production and controlled breathing) and structural (assessed using diffusion tensor tractography) connections with the laryngeal motor cortex (Simonyan et al., 2009). This may suggest a prominent role of the posterior cingulate in the control of laryngeal muscle activity during evoked coughing.

Several brain regions appear to be preferentially activated by the voluntary or cognitive components of cough and cough suppression. The anterior mid-cingulate cortex and anterior insula show increased BOLD signal for all experimental conditions, but more so after cough suppression. These regions are commonly activated in pain paradigms and could be associated with the affective aspects of unresolved irritation from the capsaicin exposure during cough suppression. Alternatively, since electrical stimulation of the anterior insula in monkeys suppresses breathing and can evoke long-lasting apnea that continues well after the cessation of stimulus (Sugar et al., 1948), this region may also play a role in respiratory-related suppression. The anterior midcingulate cortex activation is predominantly located in the upper bank of the cingulate cortex and extends to the supplementary motor area. The contiguous activation spanning from the dorsal anterior mid-cingulate cortex to a region within the supplementary motor area includes the rostral cingulate motor area and may reflect a higher order motor component of a voluntary cough initiation/suppression network, perhaps part of a behavioral response selection system (Bush et al., 2002). Indeed, the rostral cingulate motor area has well defined connections with skeletal motor pathways in the spinal cord (Dum and Strick, 1991) and (along with the prefrontal cortex) is thought to be involved in the cognitive aspects of movement such as motor selection and error detection. The negative interaction within the supplementary motor area may be associated with the volitional control of cough since it activated strongly for voluntary cough and cough suppression, but not for evoked coughing (unlike other parts of the supplementary motor area that were also activated by evoked cough). Similarly, the right inferior frontal gyrus (BA47) displayed negligible BOLD signal change after evoked cough but was strongly activated after cough suppression. This area is activated during Valsalva maneuvers and inhibits respiration when elec- trically stimulated (Smith, 1938; Henderson et al., 2003). Interestingly, the right inferior frontal gyrus, along with the pre-supplementary motor area, prefrontal cortex, cingulate, and basal ganglia, comprises an inhibitory network that activates with response inhibition during go/no-go tasks (Duann et al., 2009). Comparable activations are associated with volitional breath holding (McKay et al., 2008), supporting the notion that this circuitry is also intimately involved in respiratory and cough suppression.

\section{Medullary activations}

It is interesting to note the lack of activation of the lower brainstem during voluntary cough. This is in contrast to the medullary responses seen after evoked cough or cough suppression. The data might suggest that capsaicin is the primary determinant of medullary activations in these studies. However, this is unlikely as cough was also shown to produce medullary signal changes when analyzed independent of capsaicin. Thus, it appears that the involvement of the lower brainstem in volitional coughing may be negligible, perhaps indicative of a corticospinal cough generator. Nevertheless, concluding that voluntary cough does not involve the medulla should be made cautiously because relatively lower BOLD signal-to-noise ratios in the brainstem could limit the identification of activation. Replication of the experimental protocol with images optimized for the brainstem would provide a more reliable test of medullary activation during voluntary coughing.

\section{Conclusions}

These data provide a significant advance in understanding the neural control of coughing and support theoretical models predicting the existence of distinct excitatory and inhibitory descending influences on the cough reflex pathway. Further understanding how these networks regulate coughing may provide new possibilities for future antitussive therapies.

\section{References}

Andersson JL (1997) How to estimate global activity independent of changes in local activity. Neuroimage 6:237-244.

Baekey DM, Morris KF, Gestreau C, Li Z, Lindsey BG, Shannon R (2001) Medullary respiratory neurones and control of laryngeal motoneurones during fictive eupnoea and cough in the cat. J Physiol 534:565-581.

Birn RM, Smith MA, Jones TB, Bandettini PA (2008) The respiration response function: the temporal dynamics of fMRI signal fluctuations related to changes in respiration. Neuroimage 40:644-654.

Birn RM, Murphy K, Handwerker DA, Bandettini PA (2009) fMRI in the presence of task-correlated breathing variations. Neuroimage 47:1092-1104.

Bolser DC, Poliacek I, Jakus J, Fuller DD, Davenport PW (2006) Neurogenesis of cough, other airway defensive behaviors and breathing: a holarchical system? Respir Physiol Neurobiol 152:255-265.

Brignall K, Jayaraman B, Birring SS (2008) Quality of life and psychosocial aspects of cough. Lung 186:S55-S58.

Bush G, Vogt BA, Holmes J, Dale AM, Greve D, Jenike MA, Rosen BR (2002) Dorsal anterior cingulate cortex: a role in reward-based decision making. Proc Natl Acad Sci U S A 99:523-528.

Canning BJ, Mazzone SB, Meeker SN, Mori N, Reynolds SM, Undem BJ (2004) Identification of the tracheal and laryngeal afferent neurones mediating cough in anaesthetized guinea-pigs. J Physiol 557:543-558.

Davenport PW (2009) Clinical cough I: the urge-to-cough: a respiratory sensation. Handb Exp Pharmacol 187:263-276.

Davenport PW, Sapienza CM, Bolser DC (2002) Psychophysical assessment of the urge-to-cough. Eur Respir Rev 12:249-253.

Dicpinigaitis PV, Rauf K (1998) The influence of gender on cough reflex sensitivity. Chest 113:1319-1321.

Duann JR, Ide JS, Luo X, Li CS (2009) Functional connectivity delineates the 
inferior frontal cortex and presupplementary motor area in stop signal inhibition. J Neurosci 29:10171-10179.

Dum RP, Strick PL (1991) The origin of corticospinal projections from the premotor areas in the frontal lobe. J Neurosci 11:667-689.

Eccles R (2009) Central mechanisms IV: conscious control of cough and the placebo effect. Handb Exp Pharmacol 187:241-262.

Evans KC, Shea SA, Saykin AJ (1999) Functional MRI localisation of central nervous system regions associated with volitional inspiration in humans. J Physiol 520:383-392.

Henderson LA, Woo MA, Macey PM, Macey KE, Frysinger RC, Alger JR, Yan-Go F, Harper RM (2003) Neural responses during Valsalva maneuvers in obstructive sleep apnea syndrome. J Appl Physiol 94:1063-1074.

Hutchings HA, Morris S, Eccles R, Jawad MS (1993) Voluntary suppression of cough induced by inhalation of capsaicin in healthy volunteers. Respir Med 87:379-382.

Koritnik B, Azam S, Andrew CM, Leigh PN, Williams SC (2009) Imaging the brain during sniffing: a pilot fMRI study. Pulm Pharmacol Ther 22:97-101.

Lund TE, Madsen KH, Sidaros K, Luo WL, Nichols TE (2006) Non-white noise in fMRI: does modelling have an impact? Neuroimage 29:54-66.

Materazzi S, Nassini R, Gatti R, Trevisani M, Geppetti P (2009) Cough sensors. II. Transient receptor potential membrane receptors on cough sensors. Handb Exp Pharmacol 187:49-61.

Mazzone SB (2005) An overview of the sensory receptors regulating cough. Cough 1:2.

Mazzone SB, McLennan L, McGovern AE, Egan GF, Farrell MJ (2007) Representation of capsaicin-evoked urge-to-cough in the human brain using functional magnetic resonance imaging. Am J Respir Crit Care Med 176:327-332.

Mazzone SB, McGovern AE, Koo K, Farrell MJ (2009a) Mapping supramedullary pathways involved in cough using functional brain imaging: comparison with pain. Pulm Pharmacol Ther 22:90-96.

Mazzone SB, Reynolds SM, Mori N, Kollarik M, Farmer DG, Myers AC, Canning BJ (2009b) Selective expression of a sodium pump isozyme by cough receptors and evidence for its essential role in regulating cough. J Neurosci 29:13662-13671.

McGarvey LP, Carton C, Gamble LA, Heaney LG, Shepherd R, Ennis M, MacMahon J (2006) Prevalence of psychomorbidity among patients with chronic cough. Cough 2:4.

McKay LC, Evans KC, Frackowiak RS, Corfield DR (2003) Neural correlates of voluntary breathing in humans. J Appl Physiol 95:1170-1178.
McKay LC, Adams L, Frackowiak RS, Corfield DR (2008) A bilateral cortico-bulbar network associated with breath holding in humans, determined by functional magnetic resonance imaging. Neuroimage 40:1824-1832.

Nishino T, Hiraga K, Mizuguchi T, Honda Y (1988) Respiratory reflex responses to stimulation of tracheal mucosa in enflurane-anesthetized humans. J Appl Physiol 65:1069-1074.

Ohi Y, Yamazaki H, Takeda R, Haji A (2004) Phrenic and iliohypogastric nerve discharges during tussigenic stimulation in paralyzed and decerebrate guinea pigs and rats. Brain Res 1021:119-127.

Ramsay SC, Adams L, Murphy K, Corfield DR, Grootoonk S, Bailey DL, Frackowiak RS, Guz A (1993) Regional cerebral blood flow during volitional expiration in man: a comparison with volitional inspiration. J Physiol 461:85-101.

Shannon R, Baekey DM, Morris KF, Li Z, Lindsey BG (2000) Functional connectivity among ventrolateral medullary respiratory neurones and responses during fictive cough in the cat. J Physiol 525:207-224.

Shima K, Aya K, Mushiake H, Inase M, Aizawa H, Tanji J (1991) Two movement-related foci in the primate cingulate cortex observed in signal-triggered and self-paced forelimb movements. J Neurophysiol 65:188-202.

Simonyan K, Saad ZS, Loucks TM, Poletto CJ, Ludlow CL (2007) Functional neuroanatomy of human voluntary cough and sniff production. Neuroimage 37:401-409.

Simonyan K, Ostuni J, Ludlow CL, Horwitz B (2009) Functional but not structural networks of the human laryngeal motor cortex show left hemispheric lateralization during syllable but not breathing production. J Neurosci 29:14912-14923.

Smith WK (1938) The representation of respiratory movements in the cerebral cortex. J Neurophysiol 1:55-68.

Sugar O, Chusid JG, French JD (1948) Second motor cortex in the monkey (Macaca mulatta). J Neuropathol Exp Neurol 7:182-189.

Vogt BA (2005) Pain and emotion interactions in subregions of the cingulate gyrus. Nat Rev Neurosci 6:533-544.

Woolrich MW, Ripley BD, Brady M, Smith SM (2001) Temporal autocorrelation in univariate linear modeling of FMRI data. Neuroimage 14:1370-1386.

Worsley KJ, Evans AC, Marrett S, Neelin P (1992) A three-dimensional statistical analysis for CBF activation studies in human brain. J Cereb Blood Flow Metab 12:900-918. 